Folia phoniat. 1969;21:476

\title{
IALP News
}

Consultative status with WHO, UNESCO and UNICEF

Emil Froeschels, M. D., has been elected a member of The Royal Society for the Promotion of Health, London.

Committees Comíté d'Audiologíe Appliquée

La reunion du Comité d'Audiologie de ГIALP s'est tenue le 12 novembre 1969 à Besançon. Le

Dr C. Croatto-Martínollí, Secretaire, fit un expose sur le travail fourni et les difficultés rencontrées dans Taccomplissement de ce dernier.

Au cours de cette rencontre on décida d'adopter Tappellation «Comité d'Audiologie Appliquée » pour mieux syrithétiser les activités de ce dernier. Le but du Comité est $\Gamma$ étude de Гaudition dans les domaine pathologique et la réadaptation de la communication humaine. Pour faciliter les déplacements et la prise de contact entre les membres, on décida de créer des sections continentales.

La section européenne se compose de M. le Dr C. Croatto-Martínollí (Secretaire), de MM. les Drs Borel-Maíssony, Decroíx, Graf, D. Hennebert, Holm, Lafon, De Reyníer, Sedlacek et Zíegler. Elle sera complétée par d'autres personnalités dont on attend encore la réponse affirmative.

Affiliated Societies

Belgísche Beroepsvereníging voor Logopedisten Liste des Membres du Conseil

d'administration. President: M. Rodts, Secretaire: M. Mertens, Secretaire adj.: Mme LageyChantraín, Trésorier: M. Mertens. Res-ponsable pour les contacts internationaux: M. de Smedt, rue de la Blanchisserie 34, Malínes, Belgique. Responsable pour les publications: M. Síntubín et M. Smets. Responsable pour les contacts avec les autres associations: M. Rodts et M. van Kouteren. 